\title{
THE PRIMARY STRUCTURE OF CLOSTRIDIUM PERFRINGENS FERREDOXIN
}

\author{
YASUHIDE SEKI,* SACHIKO SEKI, AND MAKOTO ISHIMOTO ${ }^{\dagger}$ \\ Department of Chemical Microbiology, Faculty of Pharmaceutical Sciences, \\ Hokkaido University, Kita-ku, Sapporo, Hokkaido 060, Japan
}

(Received May 8, 1989)

\begin{abstract}
The complete amino acid sequence of ferredoxin from Clostridium perfringens was established by a combination of Edman degradation and protease digestion. The sequence was: AYKILDTCVSCGACAAECPVDAISQGDTQFVIDADTCIDCGNCANVCPVGAPVQE. It shows a high degree of similarity to Clostridium pasteurianum ferredoxin $(89 \%$ homology), and its residual conversions are located at the non-conservative sites of the clostridial-type ferredoxins: at 6 sites, discrete except for the last two, all along the amino(N)-terminal half of the chain, while none at the carboxyl(C)-terminal half (residue numbers from 30 to 55). The occurrence of the 8 cysteine residues at the conservative sites strongly suggested the presence of the conventional two $[4 \mathrm{Fe}-4 \mathrm{~S}]$ clusters in the molecule.
\end{abstract}

Bacterial ferredoxins are iron-sulfur proteins with low molecular weights of 6,000 to $15,000(6)$. Of these, the first and best characterized is that from an anaerobic bacterium Clostridium pasteurianum (5). The clostridial-type ferredoxin consists of 55 amino acid residues, 8 iron atoms, and 8 acid-labile sulfurs per molecule of the protein. It contains 8 cysteine residues and has two [4Fe-4S] clusters.

Ferredoxin acts as an electron donor for nitrate reductase in Clostridium perfringens (9). In a previous paper (10), we tentatively postulated that a ferredoxin isolated from the bacterium contained a single [4Fe-4S] cluster instead of the two, based on the evaluations of four atoms of iron, four atoms of acid-labile sulfur, and six half-cystine residues per molecule of the protein. In this paper we report primary structure of $C$. perfringens ferredoxin and propose the presence of two $[4 \mathrm{Fe}-4 \mathrm{~S}]$ clusters in the molecule.

* Address reprint requests to: Dr. Yasuhide Seki, Department of Chemical Microbiology, Faculty of Pharmaceutical Sciences, Hokkaido University, Kita-ku, Sapporo, Hokkaido 060, Japan.

$\dagger$ Present address: Sengoku 3-37-10, Bunkyo-ku, Tokyo 112, Japan.

Abbreviations: PE-Fd, pyridylethyl-apoferredoxin; N-, amino; and C-, carboxyl. 


\section{MATERIALS AND METHODS}

Materials. Clostridium perfringens was harvested from a GAM culture supplemented with nitrate (11), and ferredoxin was isolated from the cells as previously described (10). Ferredoxin was further purified by HPLC (CCPD system, Tosoh) on a TSK-Phenyl-5PW RP column $(0.46 \times 7.5 \mathrm{~cm})$ with a linear concentration gradient of from 10 to $90 \%(\mathrm{v} / \mathrm{v})$ acetonitrile containing $0.05 \%$ trifluoroacetic acid, monitored by the absorbance at $220 \mathrm{~nm}$. It was finally purified by reverse-phase HPLC on a TSK-ODS-120T column $(0.46 \times 25 \mathrm{~cm})$ in $0.05 \%$ trifluoroacetic acid with a linear concentration gradient from 10 to $36 \%$ acetonitrile.

Staphylococcal V8 protease [EC 3.4.21.19] was purchased from Boehringer Mannheim GmbH.

Fragmentation of ferredoxin. The ferredoxin was pyridylethylated with 4-vinylpyridine (4) and the pyridylethyl-apoferredoxin (PE-Fd) was isolated by HPLC with a Phenyl-5PW column and purified on an ODS-120T column. To the purified PE-Fd (about $5 \mathrm{nmol}$ ) dried under reduced pressure was added $96 \mu \mathrm{l}$ of $50 \mathrm{~mm}$ potassium phosphate buffer, $\mathrm{pH} 7.8$, and $4 \mu \mathrm{l}$ of aqueous staphylococcal $\mathrm{V} 8$ protease solution $(50 \mathrm{ng} / \mu \mathrm{l})$, and the mixture was incubated for $12 \mathrm{~h}$ at $37^{\circ} \mathrm{C}$. After further addition of $10 \mu \mathrm{l}$ of the V8 protease solution and another $9 \mathrm{~h}$ of incubation, the digest was added with $0.9 \mathrm{ml}$ of the solution composed of $0.05 \%$ trifluoroacetic acid and $10 \%$ acetonitrile. The resulting peptides were separated on an ODS-120T column by a gradient of acetonitrile from 10 to $45 \%$.

Amino acid analysis. The amino acid compositions of PE-Fd and derived peptides were determined with a Hitachi-835 autoanalyzer system after 14-16 h hydrolysis with $6 \mathrm{~N} \mathrm{HCl}$. The $o$-phthalaldehyde method (3) was employed when the amounts of the subjected samples were present only in an order of pmol.

The N-terminal sequences of the proteins and derivative peptides were determined by an automated protein sequencer (Applied Biosystems 477A), amino acids being determined as the phenylthiohydantoin-derivatives.

\section{RESULTS AND DISCUSSION}

The complete amino acid sequence of the ferredoxin from $C$. perfringens is shown in Fig. 1. The amino acid composition calculated from the sequence agreed

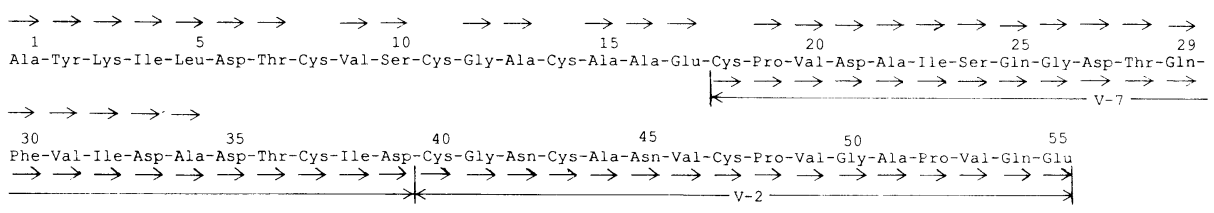

Fig. 1. The amino acid sequence of $C$. perfringens ferredoxin.

$\mathrm{V}$ - refers to peptides derived by $\mathrm{V} 8$ protease digestion and the additional numbering indicates the order of elution by HPLC (see Fig. 2). The arrows $(\rightarrow)$ represent the results of automated Edman degradation. 


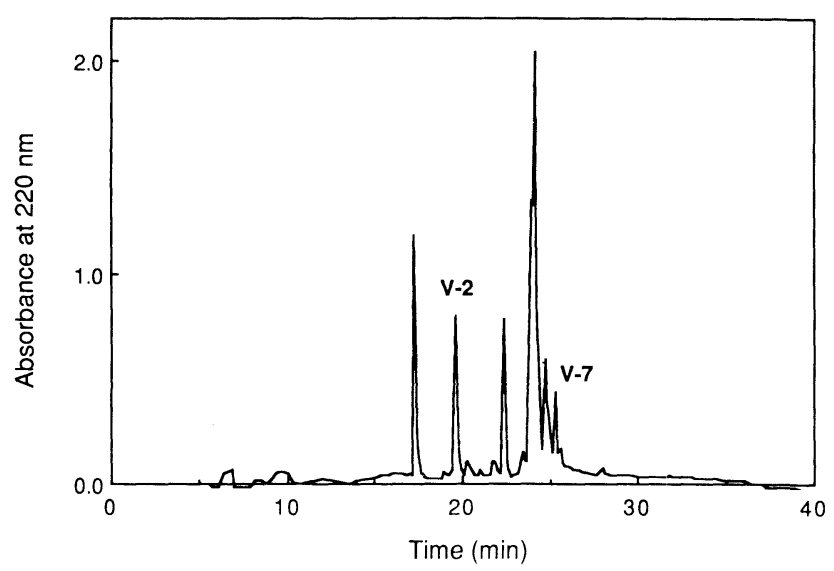

Fig. 2. The elution pattern of the V8 protease fragments of PE-Fd.

The chromatographic conditions are described in MATERIALS AND METHODS.

Table 1. Amino acid compositions of peptides obtained by V8 protease digestion.

\begin{tabular}{lrr} 
& V-2 & V-7 \\
\hline Cys & $(3)$ & $(2)$ \\
Asp & $2.1(2)$ & $4.5(5)$ \\
Thr & & $1.4(2)$ \\
Ser & & $1.0(1)$ \\
Glu & $1.9(2)$ & $1.8(2)$ \\
Gly & $2.2(2)$ & $1.4(1)$ \\
Ala & $1.8(2)$ & $1.6(2)$ \\
Val & $2.7(3)$ & $1.5(2)$ \\
Ile & & $2.3(3)$ \\
Phe & & $1.2(1)$ \\
Pro & $1.3(2)$ & $0.7(1)$ \\
& & $18-39$ \\
Position & $40-55$ & \\
\hline
\end{tabular}

Values in parentheses are deduced from sequences.

fairly well with that determined previously (10). Since the close resemblance of the composition with that of $C$. pasteurianum ferredoxin suggested also a very close resemblance in their primary structure, the construction of the final sequence relied largely on the alignments of the peptides to fit the corresponding sequence of $C$. pasteurianum ferredoxin.

The amino acid sequence of the $\mathrm{N}$-terminal region of $C$. perfringens ferredoxin as isolated could be determined up to the 34 th residue by automated Edman degradation except those corresponding to cysteine residues in $C$. pasteurianum ferredoxin. Partial digestion of PE-Fd with $\mathrm{V} 8$ protease at $\mathrm{pH} 7.8$ for $21 \mathrm{~h}$ gave more than 12 fractions on HPLC (Fig. 2). Seven major fractions were analyzed for 
their amino acid compositions. Two of them, the one eluted as the next to the front peak fraction (second peak, designated as V-2) and the other as the lattermost peak (designated as V-7) gave compositions appropriate for sequencing in the light of known sequence of $C$. pasteurianum ferredoxin (Table 1). The V-7 fragment enabled us to sequence from the 18th to 39 th residue, accompanied by an overlap with the sequence from the $\mathrm{N}$-terminus of the ferredoxin. The V-2 fragment consisted of residues from the 40th up to the C-terminus. The rest of the fragments were not subjected to the sequence analysis.

A molecular weight of ferredoxin-apoprotein from C. perfringens was calculated to be 5,569 from the sequence, and 6,265 with the assumed two [4Fe-4S] clusters (see below).

Figure 3 shows a comparison of the sequence of ferredoxin from C.perfringens with those of two [4Fe-4S] clustered-type ferredoxins from $C$. pasteurianum (5), $C$. butyricum (2), C. acidi-urici (8), C. tartarivorum (12), C. thermosaccharolyticum (13), Clostridium sp.M-E (14), and Peptococcus aerogenes (1). At least 24 residues occurred in the identical positions. It is apparent that the sequence of $C$. perfringens ferredoxin is competent to accommodate two [4Fe-4S] clusters, with 8 cysteine residues in the identical positions as the other two [4Fe-4S]-type ferredoxins. Our conclusion here is that this ferredoxin belongs to the conventional clostridial-type ferredoxin. Our previous determination of the contents of iron, labile sulfur, and cysteine residue might have resulted from an underestimation. Or more likely, $C$. perfringens ferredoxin may not be stable enough to maintain intact iron-sulfur cluster(s) during the purification procedure and half of the iron and labile-sulfur atoms may have been lost at the final step of the preparation. This accounts very well for the lower values obtained for the molar extinction coefficient at $390 \mathrm{~nm}$ and for the specific

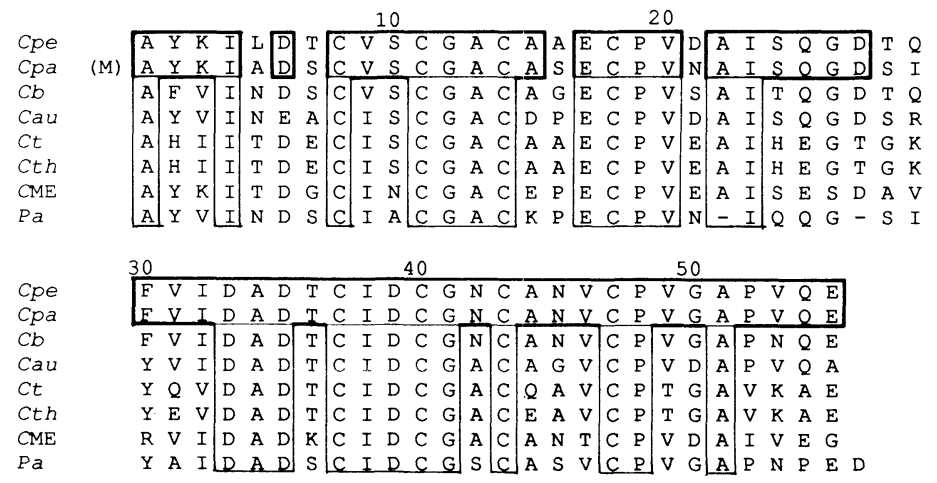

Fig. 3. Comparison of ferredoxin from Clostridium perfringens $(C p e)$ with clostridial-type ferredoxins.

$C p a, C b, C a u, C t, C t h, C \mathrm{ME}$, and $P a$ are abbreviations for $C$. pasteurianum, $C$. butyricum, C. acidi-urici, C. tartarivorum, C. thermosaccharolyticum, Clostridium species $\mathrm{ME}$, and Peptococcus aerogenes, respectively. The residues that were identical (including the gaps) within all eight sequences are boxed, and the residues identical in $C$.perfringens and $C$. pasteurianum ferredoxins are boxed with bold lines. 
activity as electron donor for nitrate reductase with $C$. perfringens ferredoxin than those with $C$. pasteurianum ferredoxin (10).

Of the seven ferredoxins compared here, $C$. perfringens ferredoxin is most closely related to $C$. pasteurianum ferredoxin. Between these two strains, 6 residues out of the 55 varied only in the $\mathrm{N}$-terminal halves of the ferredoxin molecules. The latter C-terminal halves (residues 30-55) were identical. This is the solitary exception among Clostridia with leucine residue at position 5 (and Thr at 7) because even though $C$. butyricum ferredoxin has a $\mathrm{C}$-half almost identical to the $C$. pasteurianum ferredoxin, there exists one replacement at position 53 (Asn in C. butyricum ferredoxin instead of Val in C. pasteurianum ferredoxin). It is interesting that the "connector" (residues at position 27-29 in this case), which plays an important role other than just providing an arm for the connection of the first (residues 1-26) and the second (30-55) units in the functional ferredoxin(7), has a sequence of Asp-Thr-Gln (27-29) in the $C$. perfringens ferredoxin just as in $C$. butyricum ferredoxin. On the other hand it is Asp-Ser-Ile (27-29) in C. pasteurianum ferredoxin. These are the only successive variations observed between $C$. perfringens ferredoxin and $C$. pasteurianum ferredoxin (the other 4 variations occur not successively but dispersively).

We thank Miss K. Sasaki at the Center for Instrumental Analysis in Hokkaido University for amino acid analysis.

\section{REFERENCES}

1) Adman, E. T., Sieker, L. C., and Jensen, L. H., The structure of a bacterial ferredoxin. J. Biol. Chem., 248, 3987-3996 (1973).

2) Benson, A. M., Mower, H. M., and Yasunobu, K. T., The amino acid sequences of Clostridium butyricum ferredoxin. Arch. Biochem. Biophys., 121, 563-575 (1967).

3) Benson, R. B. and Hare, P. E., $o$-Phthalaldehyde: Fluorogenic detection of primary amines in the picomole range. Comparison with fluorescamine and ninhydrin. Proc. Natl. Acad. Sci. U.S.A., 72, 619-622 (1975).

4) Freidman, M., Krull, L. H., and Cavins, J. F., The chromatographic determination of cystine and cysteine residues in proteins as $S$ - $\beta$-(4-pyridylethyl)cysteine. J. Biol. Chem., 245, 3868-3871 (1970).

5) Graves, M. C., Mullenbach, G. T., and Rabinowitz, J. C., Cloning and nucleotide sequence determination of the Clostridium pasteurianum ferredoxin gene. Proc. Natl. Acad. Sci. U.S.A., 82, 1653-1657 (1985).

6) Malikin, R., 1. The chemical properties of ferredoxins. In Iron-Sulfur Proteins, Vol. II, ed. by Lovenberg, W., Academic Press, New York and London (1973), p. 1-26.

7) Otaka, E. and Ooi, T., Examination of protein sequence homologies: IV. Twenty-seven bacterial ferredoxins. J. Mol. Evol., 26, 257-260 (1987).

8) Rall, S. C., Bolinger, R. E., and Cole, R. D., The amino acid sequence of ferredoxin from Clostridium acidi-urici. Biochemistry, 7, 2486-2496 (1969).

9) Seki-Chiba, S. and Ishimoto, M., Studies on nitrate reductase of Clostridium perfringens. I. Purification, some properties, and effect of tangustate on its formation. J. Biochem., 82, 1663-1671 (1977).

10) Seki, S., Hagiwara, M., Kudo, K., and Ishimoto, M., Studies on nitrate reductase of Clostridium perfringens. II. Purification and some properties of ferredoxin. J. Biochem., 85, 833-838 (1979). 
11) Seki, S., Higo, K., and Ishimoto, M., Studies on nitrate reductase of Clostridium perfringens. III. Comparison of nitrate reductase from five strains of C. perfringens. J. Gen. Appl. Microbiol., 28, 541-550 (1982).

12) Tanaka, M., Haniu, M., Matsueda, G., Yasunobu, K. T., Himes, R. H., Akagi, J. M., Barnes, E. M., and Devanathan, T., The primary structure of the Clostridium tartarivorum ferredoxin, a heat-stable ferredoxin. J. Biol. Chem., 246, 3953-3960 (1971).

13) Tanaka, M., Haniu, M., Yasunobu, K. T., Himes, R. H., and Akagi, J. M., The primary structure of the Clostridium thermosaccharolyticum ferredoxin, a heat-stable ferredoxin. J. Biol. Chem., $\mathbf{2 4 8 ,}$ 5215-5217 (1973).

14) Tanaka, M., Haniu, M., Yasunobu, K. T., Jones, J. B., and Stadtman, T. C., Amino acid sequence determination of the Clostridium M-E ferredoxin and a comment on the role of the aromatic residues in the clostridial ferredoxins. Biochemistry, 12, 5284-5289 (1974). 